\title{
INTEGRAL PROPERTIES OF CERTAIN CLASSES OF MULTIVALENT ANALYTIC FUNCTIONS WITH COMPLEX ORDER
}

\author{
TARIQ O. SALIM
}

\begin{abstract}
In the present paper, the generalized Komatu integral operator and the generalized Jung-Kim-Srivastava integral operator are applied to study the integral properties of two subclasses of analytic and $p$-valent functions of complex order. Some special cases (known or new) of the main theorems are indicated.
\end{abstract}

\section{Introduction}

Let $A_{p}(n)$ denote the class of functions of the form

$$
f(z)=z^{p}-\sum_{k=n+p}^{\infty} a_{k} z^{k}, \quad\left(a_{k} \geq 0 ; n, p \in \mathbb{N}\right)
$$

that are analytic and $p$-valent in the open unit disk $U=\{z: z \in \mathbb{C},|z|<1\}$.

Differentiating both sides of (1.1) $m$-times with respect to $z$, we get

$$
f^{(m)}(z)=\frac{p !}{(p-m) !} z^{p-m}-\sum_{k=n+p}^{\infty} \frac{k !}{(k-m) !} a_{k} z^{k-m}
$$

where $n, p \in \mathbb{N}, m \in \mathbb{N}_{0}=\mathbb{N} \cup\{0\} ; p>m$.

Now, let us recall the subclasses $R_{n, m}^{p}(\lambda, b)$ and $L_{n, m}^{p}(\lambda, b)$ introduced and studied recently by Srivastava and Orhan [7] and Güney and Breaz [1], which consists of functions $f(z)$ belonging to $A_{p}(n)$ and satisfying the inequality, respectively:

$$
\left|\frac{1}{b}\left(\frac{z f^{(1+m)}(z)+\lambda z^{2} f^{(2+m)}(z)}{\lambda z f^{(1+m)}(z)+(1-\lambda) f^{(m)}(z)}-(p-m)\right)\right|<1
$$

and

$$
\left|\frac{1}{b}\left(f^{(1+m)}(z)+\lambda z f^{(2+m)}(z)-(p-m)\right)\right|<p-m .
$$

where $z \in U ; p \in \mathbb{N}, m \in \mathbb{N}_{0} ; p>m ; 0 \leq \lambda \leq 1$ and $b \in \mathbb{C} \backslash\{0\}$.

2010 Mathematics Subject Classification. 30C45, 33C20.

Key words and phrases. p-valent functions, Komatu integral operator, Jung-Kim-Srivastava integral operator. 
Srivastava and Orhan [7] (see, e.g. Güney and Breaz [1]) proved the following lemmas, in which coefficient inequalities are given as necessary and sufficient conditions for functions $f(z) \in A_{p}(n)$ to belong to the classes $R_{n, m}^{p}(\lambda, b)$ and $L_{n, m}^{p}(\lambda, b)$.

Lemma 1.1. Let $f(z) \in A_{p}(n)$ be given by (1.1). Then $f(z) \in R_{n, m}^{p}(\lambda, b)$ if and only if

$$
\sum_{k=n+p}^{\infty} \frac{(k+|b|-p) k ![\lambda(k-m-1)+1]}{(k-m) !} a_{k} \leq \frac{|b| p ![\lambda(p-m-1)+1]}{(p-m) !} .
$$

Lemma 1.2. Let $f(z) \in A_{p}(n)$ be given by (1.1). Then $f(z) \in L_{n, m}^{p}(\lambda, b)$ if and only if

$$
\sum_{k=n+p}^{\infty}\left(\begin{array}{c}
k \\
m
\end{array}\right)(k-m)[\lambda(k-m-1)+1] a_{k} \leq(p-m)\left[\frac{|b|-1}{m !}\left(\begin{array}{c}
p \\
m
\end{array}\right)[\lambda(p-m-1)+1]\right] \text {. }
$$

The generalized Komatu integral operator [4], $P_{c, p}^{\delta}: A_{p}(n) \rightarrow A_{p}(n)$ and the generalized Jung-Kim-Srivastava integral operator [2], $Q_{c, p}^{\delta}: A_{p}(n) \rightarrow A_{p}(n)$ (see,e.g. [3] and [6]) are defined for $\delta>0$ and $c>-p$ as

$$
P_{c, p}^{\delta} f(z)=\frac{(c+p)^{\delta}}{\Gamma(\delta) z^{c}} \int_{0}^{z} t^{c-1}\left(\log \frac{z}{t}\right)^{\delta-1} f(t) d t
$$

and

$$
Q_{c, p}^{\delta} f(z)=\left(\begin{array}{c}
\delta+c+p-1 \\
c+p-1
\end{array}\right) \frac{\delta}{z^{c}} \int_{0}^{z} t^{c-1}\left(1-\frac{t}{z}\right)^{\delta-1} f(t) d t
$$

Note that for functions $f(z) \in A_{p}(n)$ of the form (1.1), we have

$$
P_{c, p}^{\delta} f(z)=z^{p}-\sum_{k=n+p}^{\infty}\left(\frac{c+p}{c+k}\right)^{\delta} a_{k} z^{k}
$$

and

$$
Q_{c, p}^{\delta} f(z)=z^{p}-\sum_{k=n+p}^{\infty} \frac{(c+p)_{k-p}}{(c+p+\delta)_{k-p}} a_{k} z^{k}
$$

where

$$
(\sigma)_{k}=\frac{\Gamma(\sigma+k)}{\Gamma(\sigma)}= \begin{cases}1, & (k=0, \sigma \in \mathbb{C} \backslash\{0\}) \\ \sigma(\sigma+1) \cdots(\sigma+n-1), & (k=n \in \mathbb{N}, \sigma \in \mathbb{C})\end{cases}
$$

is the Pochhammer symbol of the extended factorial function.

Now, let us define the integral operator $H_{c, p}^{\delta, \mu}: A_{p}(n) \rightarrow A_{p}(n)$ by

$$
\begin{aligned}
H(z) & =H_{c, p}^{\delta, \mu} f(z)=\mu P_{c, p}^{\delta} f(z)+(1-\mu) Q_{c, p}^{\delta} f(z) \\
& =\int_{0}^{z} \frac{t^{c-1}}{\Gamma(\delta) z^{c}}\left[\mu(c+p)^{\delta}\left(\log \frac{z}{t}\right)^{\delta-1}+(1-\mu) \frac{\Gamma(c+p+\delta)}{\Gamma(c+p)}\left(1-\frac{t}{z}\right)^{\delta-1}\right] f(t) d t
\end{aligned}
$$


for $\delta>0, c>-p, p \in \mathbb{N}$ and $0 \leq \mu \leq 1$.

Hence, in view of (1.9) and (1.10), for functions $f(z) \in A_{p}(n)$ of the form (1.1), we have

$$
H(z)=z^{p}-\sum_{k=n+p}^{\infty}\left[\mu\left(\frac{c+p}{c+k}\right)^{\delta}+(1-\mu) \frac{(c+p)_{k-p}}{(c+p+\delta)_{k-p}}\right] a_{k} z^{k}
$$

Motivated by the recent investigations by Sălăgean [5] and Güney and Breaz [1] and others, the main object of the present investigation is to establish integral properties of analytic $p$-valently functions with negative coefficients and with complex order belonging to the classes $R_{n, m}^{p}(\lambda, b)$ and $L_{n, m}^{p}(\lambda, b)$.

\section{Integral properties of the class $R_{n, m}^{p}(\lambda, b)$}

We begin by proving the following Lemma which is needed to proceed in proving the main theorems.

Lemma 2.1. Let

$$
B_{k}=B(k, c, p, \mu, \delta)=\left[\mu\left(\frac{c+p}{c+k}\right)^{\delta}+(1-\mu) \frac{(c+p)_{k-p}}{(c+p+\delta)_{k-p}}\right]
$$

for $k \geq n+p, \delta>0, c>-p, \quad p \in \mathbb{N}$ and $0 \leq \mu \leq 1$. Then $B_{k}$ is a decreasing function of $k$, and

$$
B_{k+1} \leq B_{k} \leq B_{n+p} \leq 1
$$

Proof. Since $k=n+p \geq p$ for $n \in \mathbb{N}$, then

$$
\frac{c+p}{c+k+1} \leq \frac{c+p}{c+k} \leq \frac{c+p}{c+n+p} \leq 1
$$

and

$$
\frac{(c+p)_{k+1-p}}{(c+p+\delta)_{k+1-p}}=\frac{(c+p)_{k-p}}{(c+p+\delta)_{k-p}} \frac{c+k}{c+k+\delta} \leq \frac{(c+p)_{k-p}}{(c+p+\delta)_{k-p}} \leq \frac{(c+p)_{n}}{(c+p+\delta)_{n}} \leq 1
$$

which yields (2.2).

Now we prove

Theorem 2.2. If $f(z) \in R_{n, m}^{p}(\lambda, b)$, then $H(z) \in R_{n, m}^{p}(\lambda, \gamma)$, where

$$
|\gamma|=\frac{n|b| B_{n+p}}{n+|b|\left(1-B_{n+p}\right)}
$$

and $|\gamma|<|b|$, provided that $p, n \in \mathbb{N}, m \in \mathbb{N}_{0}, \quad \delta>0, \quad b \in \mathbb{C} \backslash\{0\}, \quad c>-p, \quad 0 \leq \mu \leq 1, \quad 0 \leq \lambda \leq 1$ and $B_{n+p}$ is given by (2.1). The result is sharp. 
Proof. From Lemma (1.1) and (1.12), $H(z) \in R_{n, m}^{p}(\lambda, \gamma)$ if and only if

$$
\sum_{k=n+p}^{\infty} \frac{(k+|\gamma|-p) k !(p-m) ![\lambda(k-m-1)+1]}{(k-m) !|\gamma| p ![\lambda(p-m-1)+1]} B_{k} a_{k} \leq 1
$$

Now for $k \geq n+p$, the inequality

$$
\begin{aligned}
& \frac{(k+|\gamma|-p) k !(p-m) ![\lambda(k-m-1)+1]}{(k-m) !|\gamma| p ![\lambda(p-m-1)+1]} B_{k} \\
& \quad \leq \frac{(k+|b|-p) k !(p-m) ![\lambda(k-m-1)+1]}{(k-m) !|b| p ![\lambda(p-m-1)+1]}
\end{aligned}
$$

implies (2.4), since $f \in R_{n, m}^{p}(\lambda, b)$ and satisfies (1.5). Hence

$$
\frac{(k+|\gamma|-p) B_{k}}{|\gamma|} \leq \frac{(k+|b|-p)}{|b|}
$$

which yields

$$
|\gamma| \geq \frac{(k-p)|b| B_{k}}{(k-p)+|b|\left(1-B_{k}\right)}
$$

Now, we show that $|\gamma|$ is a decreasing function of $k, k \geq n+p$. Indeed, let

$$
h(k)=\frac{(k-p)|b| B_{k}}{(k-p)+|b|\left(1-B_{k}\right)}
$$

So, in view of Lemma (2.1), we have

$$
\begin{aligned}
h(k+1)-h(k) & =\frac{(k+1-p)|b| B_{k+1}}{(k+1-p)+|b|\left(1-B_{k+1}\right)}-\frac{(k-p)|b| B_{k}}{(k-p)+|b|\left(1-B_{k}\right)} \\
& =\frac{(k+1-p)(k-p)|b|\left(B_{k+1}-B_{k}\right)+(k+1-p)|b|^{2} B_{k+1}\left(1-B_{k}\right)-(k-p)|b|^{2} B_{k}\left(1-B_{k+1}\right)}{\left[(k+1-p)+|b|\left(1-B_{k+1}\right)\right]\left[(k-p)+|b|\left(1-B_{k}\right)\right]} \\
& \leq \frac{(k+1-p)(k-p)|b|\left(B_{k+1}-B_{k}\right)+(k+1-p)|b|^{2}\left(B_{k+1}-B_{k}\right)}{\left[(k+1-p)+|b|\left(1-B_{k+1}\right)\right]\left[(k-p)+|b|\left(1-B_{k}\right)\right]} \\
& \leq 0 .
\end{aligned}
$$

So $h(k)$ is a decreasing function of $k$ and we have

$$
|\gamma(p, k, c, b, \mu, \delta)| \leq|\gamma|=|\gamma(p, p+n, c, b, \mu, \delta)| ; \quad k \geq n+p
$$

Thus (2.3) is proved, and in view of Lemma (2.1), $B_{n+p} \leq 1$ directly implies $|\gamma|<|b|$.

Now , the result is sharp because

$$
H_{c, p}^{\delta, \mu}\left(f_{b}\right)=f_{\gamma}
$$


where

$$
f_{b}(z)=z^{p}-\frac{|b| p !(n+p-m)[\lambda(p-m-1)+1]}{(p-m) !(n+p) !(n+|b|)[\lambda(p-m-1)+1]} z^{n+p}
$$

and

$$
f_{\gamma}(z)=z^{p}-\frac{|\gamma| p !(n+p-m)[\lambda(p-m-1)+1]}{(p-m) !(n+p) !(n+|\gamma|)[\lambda(p-m-1)+1]} z^{n+p}
$$

are extremal functions of $R_{n, m}^{p}(\lambda, b)$ and $R_{n, m}^{p}(\lambda, \gamma)$, respectively.

Indeed, we have

$$
H_{c, p}^{\delta, \mu}\left(f_{b}\right)(z)=z^{p}-\frac{|b| p !(n+p-m)[\lambda(p-m-1)+1]}{(p-m) !(n+p) !(n+|b|)[\lambda(p-m-1)+1]} B_{n+p} z^{n+p}
$$

Thus in comparison with (2.11), we deduce

$$
\frac{|\gamma|}{n+|\gamma|}=\frac{|b|}{n+|b|} B_{n+p}
$$

which implies (2.8).

Setting $\mu=1$ and $\mu=0$, respectively in Theorem (2.2), we get the following corollaries:

Corollary 2.3. If $f(z) \in R_{n, m}^{p}(\lambda, b)$, then $P_{c, p}^{\delta} f(z) \in R_{n, m}^{p}\left(\lambda, \gamma_{1}\right)$, where

$$
\left|\gamma_{1}\right|=\frac{n|b|(c+p)^{\delta}}{n(c+n+p)^{\delta}+|b|\left[(c+n+p)^{\delta}-(c+p)^{\delta}\right]}
$$

and $\left|\gamma_{1}\right|<|b|$, provided that $p, n \in \mathbb{N}, m \in \mathbb{N}_{0}, \quad \delta>0, \quad b \in \mathbb{C} \backslash\{0\}, \quad c>-p$ and $0 \leq \lambda \leq 1$. The result is sharp.

Corollary 2.4. If $f(z) \in R_{n, m}^{p}(\lambda, b)$, then $Q_{c, p}^{\delta} f(z) \in R_{n, m}^{p}\left(\lambda, \gamma_{2}\right)$, where

$$
\left|\gamma_{2}\right|=\frac{n|b|(c+p)_{n}}{n(c+p+\delta)_{n}+|b|\left[(c+p+\delta)_{n}-(c+p)_{n}\right]}
$$

and $\left|\gamma_{2}\right|<|b|$, provided that $p, n \in \mathbb{N}, m \in \mathbb{N}_{0}, \quad \delta>0, \quad b \in \mathbb{C} \backslash\{0\}, \quad c>-p$ and $0 \leq \lambda \leq 1$. The result is sharp.

Setting $\delta=1$ in either (2.14) or (2.15), we get the result recently obtained by Güney and Breaz [1, Theorem 1]. Also, if we put $\delta=1, \quad p=1, \quad m=0, \quad b=1-\alpha$ and $\gamma=1-\beta$, we obtain the result established by Sălăgean [5]. Several special cases can be obtained by specifying the parameters in Theorem (2.2). 
3. Integral properties of the class $L_{n, m}^{p}(\lambda, b)$

Theorem 3.1. If $f(z) \in L_{n, m}^{p}(\lambda, b)$, then $H(z) \in L_{n, m}^{p}(\lambda, \beta)$, where

$$
|\beta|=(|b|-1) B_{n+p}+\frac{p !}{(p-m) !}[\lambda(p-m-1)+1]\left(B_{n+p}-1\right)+1
$$

and $|\beta|<|b|$, provided that $p, n \in \mathbb{N}, m \in \mathbb{N}_{0} \delta>0, \quad b \in \mathbb{C} \backslash\{0\}, c>-p, 0 \leq \mu \leq 1,0 \leq \lambda \leq 1$ and $B_{n+p}$ is given by (2.1). The result is sharp.

Proof. Following similar steps to that in the proof of Theorem (2.2), we can get the result

Setting $\mu=1$ and $\mu=0$, respectively in Theorem 3.1, we get the following corollaries:

Corollary 3.2. If $f(z) \in L_{n, m}^{p}(\lambda, b)$, then $P_{c, p}^{\delta} f(z) \in L_{n, m}^{p}\left(\lambda, \beta_{1}\right)$, where

$$
\left|\beta_{1}\right|=(|b|-1)\left(\frac{c+p}{c+n+p}\right)^{\delta}+\frac{p !}{(p-m) !}[\lambda(p-m-1)+1]\left(\left(\frac{c+p}{c+n+p}\right)^{\delta}-1\right)+1
$$

and $\left|\beta_{1}\right|<|b|$, provided that $p, n \in \mathbb{N}, m \in \mathbb{N}_{0}, \quad \delta>0, \quad b \in \mathbb{C} \backslash\{0\}, \quad c>-p$ and $0 \leq \lambda \leq 1$. The result is sharp.

Corollary 3.3. If $f(z) \in L_{n, m}^{p}(\lambda, b)$, then $Q_{c, p}^{\delta} f(z) \in L_{n, m}^{p}\left(\lambda, \beta_{2}\right)$, where

$$
\left|\beta_{2}\right|=(|b|-1) \frac{(c+p)_{n}}{(c+p+\delta)_{n}}+\frac{p !}{(p-m) !}[\lambda(p-m-1)+1]\left(\frac{(c+p)_{n}}{(c+p+\delta)_{n}}-1\right)+1
$$

and $\left|\beta_{2}\right|<|b|$, provided that $p, n \in \mathbb{N}, m \in \mathbb{N}_{0}, \quad \delta>0, \quad b \in \mathbb{C} \backslash\{0\}, \quad c>-p$ and $0 \leq \lambda \leq 1$. The result is sharp.

Setting $\delta=1$ in either (3.2) or (3.3), we get the result recently obtained by Güney and Breaz [1, Theorem 2]. More particular results can be obtained by specifying the parameters in Theorem 3.1.

\section{References}

[1] H. Ö. Güney, D. Breaz, Integral properties of some families of multivalent functions with complex order, Studia Univ. "Babeş-Bolyai", Mathematica LIV 1 (2009), 101-106.

[2] I. B. Jung, Y. C. Kim, H. M. Srivastava, The Hardy space of analytic functions associated with certain oneparameter families of integral operators, J. Math. Anal. Appl. 176 (1993), 138-147.

[3] S. M. Khairnar, M. More, On a subclass of multivalent $\beta$-uniformly starlike and convex functions defined by a linear operator, IAENG Int. J. Appl. Math. 39:3, IJAM-39-06 (2009).

[4] Y. Komatu, On analytic prolongation of a family of operators, Mathematica (Cluj) 3255 (1990), 141-145.

[5] G. S. Sălăgean, Integral properties of certain classes of analytic functions with negative coefficients, Int. J. Math. Math. Sci., 1 (2005), 125-131. 
[6] T. O. Salim, Certain classes of multivalent functions defined by a fractional differential operator, General Mathematics, 19(3) (2011), 75-84.

[7] H. M. Srivastava and H. Orhan, Coefficient inequalities and inclusion relations for some families of analytic and multivalent functions, Appl. Math. Lett., 206 (2007), 686-691

Department of Mathematics, Al-Azhar University-Gaza, P.O. Box 1277, Gaza, Palestine.

E-mail: trsalim@yahoo.com; t.salim@alazhar.edu.ps 